\title{
Biomedical Applications of Multi-Material Phase Retrieval in Propagation-Based Phase-Contrast Imaging
}

\author{
$\underline{\text { Ilian Häggmark }}{ }^{1, *}$, William Vågberg ${ }^{1}$, Hans M. Hertz $^{1}$, and Anna Burvall ${ }^{1}$ \\ 1. Dept. of Applied Physics, KTH Royal Institute of Technology, Stockholm, Sweden. \\ * Corresponding author, ilian.haggmark@biox.kth.se
}

In propagation-based phase-contrast imaging the measured quantity is proportional to the second-order derivative of the phase shift induced by the object. This makes the techniques more sensitive to high spatial frequencies and low-absorbing materials than conventional X-ray imaging. To obtain an image more similar to the object and to get quantitative values it is, however, necessary to retrieve the original phase shift from its second-order derivative. This process, phase retrieval, can in simple cases, such as a single-material object in air, be done with robust and quantitative methods [1]. For multi-material samples it is a challenge, especially for polychromatic laboratory sources. We have previously described theoretical aspects and performance of two multi-material methods [2]. Here we demonstrate two examples of biomedical samples that benefit from this type of more advanced phase retrieval.

In practice, phase retrieval can be considered as a conversion of the edge enhancement inherent to propagation-based phase-contrast images to image contrast. A filter procedure removes an edge while increasing the contrast-to-noise ratio. For a multi-material sample several different interfaces exist and the intensity of each edge vary. Applying a simple filter to this sample will either result in remaining edge enhancement at some interface or too much removal (blurring). To avoid this, multi-material methods apply phase-retrieval filters locally in the image. To modify an image locally, 3D data is required and these methods are hence limited to tomographic imaging. Of the methods compared in [2] the newer method by Ullherr and Zabler [3] was deemed faster, simpler to use and slightly more accurate. The method is performed in five steps: 1) conventional phase retrieval on raw projection images that leaves remaining edge enhancement, 2) tomographic reconstruction, 3) segmentation of the 3D image to isolate the parts with remaining edge enhancement, 4) secondary phase retrieval on part of the 3D image, 5) merging of the 3D image. The resulting images are qualitatively accurate in the case of polychromatic radiation and quantitatively in the case of monochromatic radiation.

Figure 1 shows a sample of breast tissue where calcifications and adipose tissue is visible [4]. Highly absorbing parts like bone and calcifications require in general little phase retrieval compared to soft tissue. Phase retrieval optimized for soft tissue will therefore severely blur the highly absorbing region and destroy structural features. As noted in [4] a single-material phase retrieval is unable to retrieve all features well. A multi-material phase retrieval is therefore necessary to simultaneously observe both the highly absorbing regions and the soft tissue. Multi-materials methods furthermore enable studying the soft tissue close to the more absorbing region, something that would be difficult if the bright signal from the bone/calcifications was smeared out in a phase retrieval for soft tissue.

Figure 2 shows an axial slice of human coronary artery infested with calcium rich plaque, leading to obstruction of blood flow [5]. Heart disease being one of the major causes of death in the world, these plaques and surrounding tissue are of high medical interest. Like the sample above, the more absorbing parts of the image will blur and affect neighboring tissue if simple phase retrieval is aimed at the cells. Using the multi-material method, small variations in the soft tissue can be studied also close to the calcified plaque. 
The two displayed examples show that multi-material methods are important for phase retrieving an image in its entirety and to retrieve low-absorbing materials spatially close to highly absorbing ones [6].

\section{References:}

[1] A Burvall et al, Opt. Express 19 (2011) p. 10359.

[2] I Häggmark et al, Opt. Express 25 (2017) p. 33543.

[3] M Ullherr and S Zabler, Opt. Express 23 (2015) p. 32718.

[4] T Zhou et al, Opt. Express 21 (2013) p. 30183.

[5] W Vågberg et al, "Cellular-resolution 3D virtual histology of human coronary arteries using x-ray phase tomography", submitted.

[6] The authors acknowledge funding from the Swedish Research Council and the Wallenberg Foundation.

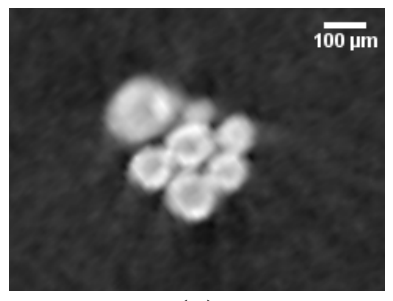

(a)

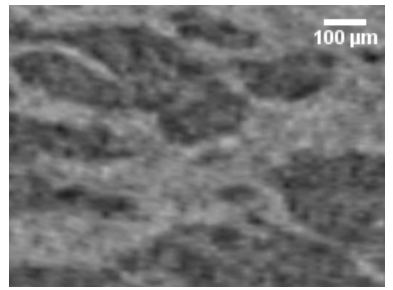

(d)

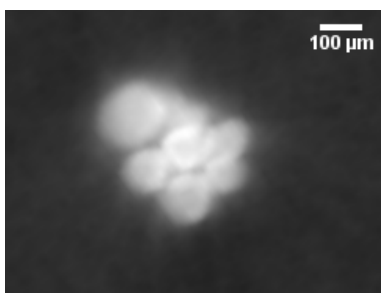

(b)

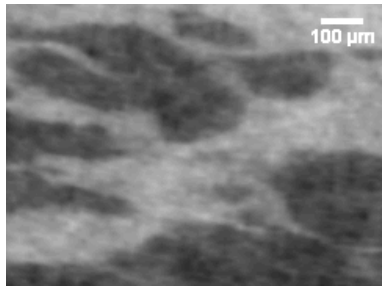

(e)

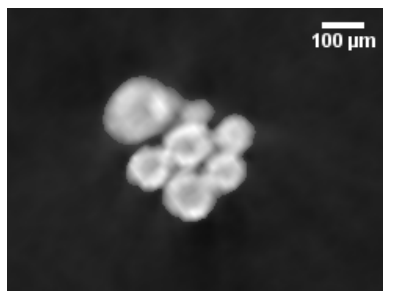

(c)

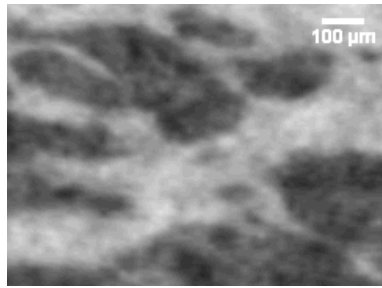

(f)

Figure. 1. Calcifications (a-c) and adipose tissue (d-f) from a single slice in a breast tissue sample. The phase retrieval used is: incomplete single-material ( $a$ and d), full single-material ( $b$ and e), and multi-material (c and f). (a-b) and (d-e) are adapted from [4].

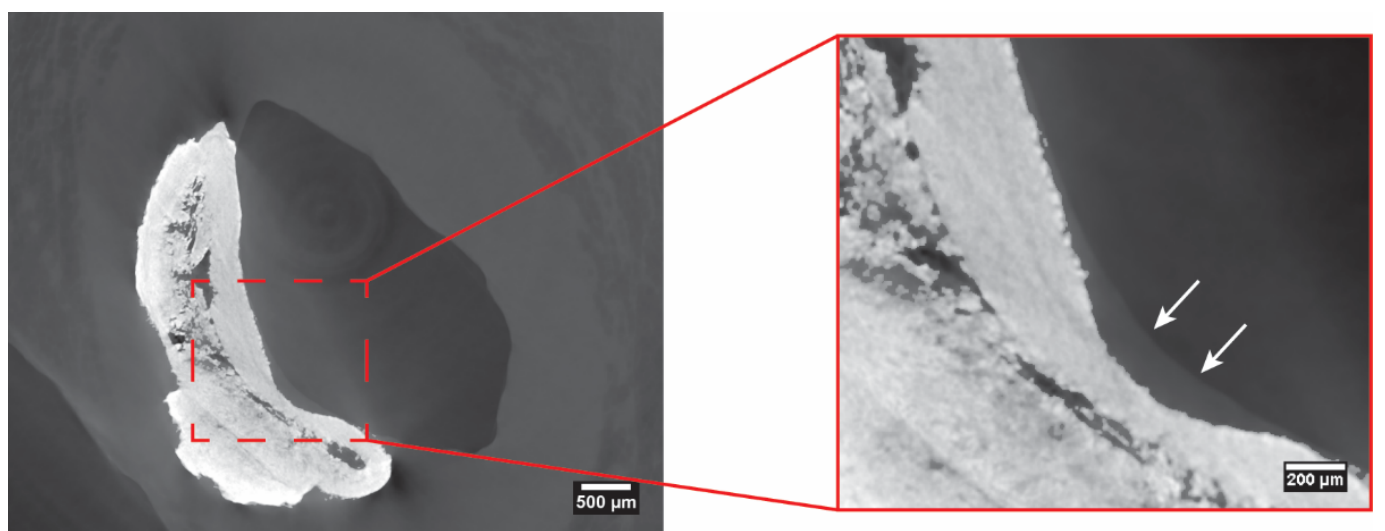

(a)

(b)

Figure. 2. (a) Axial slice of human coronary artery. The center is the lumen and the bight region the calcified plaque. (b) The thin soft tissue region between plaque (bright) lumen (dark) is retrieved well. 